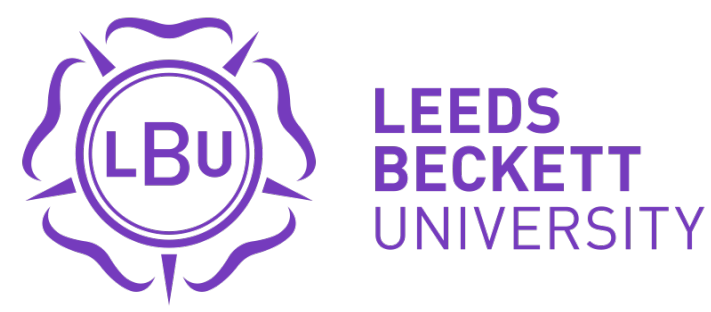

Citation:

Vajpayee, $\mathrm{V}$ and Becerra, $\mathrm{V}$ and Bausch, $\mathrm{N}$ and Banerjee, S and Deng, $\mathrm{J}$ and Shimjith, SR and Arul, AJ (2020) Disturbance Observer-based Subspace Predictive Control of a Pressurized Water-type Nuclear Reactor. 2020 7th International Conference on Control, Decision and Information Technologies (CoDIT). pp. 1012-1017. ISSN 2576-3555 DOI: https://doi.org/10.1109/CoDIT49905.2020.9263802

Link to Leeds Beckett Repository record:

https://eprints.leedsbeckett.ac.uk/id/eprint/6983/

Document Version:

Article (Accepted Version)

(C) 2020 IEEE. Personal use of this material is permitted. Permission from IEEE must be obtained for all other uses, in any current or future media, including reprinting/republishing this material for advertising or promotional purposes, creating new collective works, for resale or redistribution to servers or lists, or reuse of any copyrighted component of this work in other works.

The aim of the Leeds Beckett Repository is to provide open access to our research, as required by funder policies and permitted by publishers and copyright law.

The Leeds Beckett repository holds a wide range of publications, each of which has been checked for copyright and the relevant embargo period has been applied by the Research Services team.

We operate on a standard take-down policy. If you are the author or publisher of an output and you would like it removed from the repository, please contact us and we will investigate on a case-by-case basis.

Each thesis in the repository has been cleared where necessary by the author for third party copyright. If you would like a thesis to be removed from the repository or believe there is an issue with copyright, please contact us on openaccess@leedsbeckett.ac.uk and we will investigate on a case-by-case basis. 


\title{
Disturbance Observer-based Subspace Predictive Control of a Pressurized Water-type Nuclear Reactor
}

\author{
Vineet Vajpayee, Victor Becerra, Nils Bausch, Shohan Banerjee, Jiamei Deng, S. R. Shimjith, A. John Arul
}

\begin{abstract}
This work presents a disturbance observer-based predictive control strategy using a subspace matrix structure. The aim is to improve the capability of classical predictive controllers in handling external disturbances. A subspace-based predictive controller is designed directly from measurements. Then, a disturbance observer is designed using subspace matrices to estimate the external disturbance. Both of the designs are integrated using a feed-forward plus feed-back strategy to form the proposed control strategy. The proposed scheme is tested with a simulated model of a pressurized water nuclear reactor. The effectiveness of the proposed technique is demonstrated for two different load-following operations. Further, a quantitative analysis is performed to analyse the control performance of the proposed approach.
\end{abstract}

\section{INTRODUCTION}

Nuclear power plants are complex, highly-constrained, and non-linear systems. Control of a nuclear power plant during load-following mode of operation is a challenging task due to parameter variations caused by fuel burn-up, internal reactivity feedbacks, modelling uncertainties, and unknown disturbances. System parameters associated with reactor core, thermal-hydraulics, and internal reactivity feedbacks differ significantly with operating power levels. Furthermore, the routine load cycles can significantly degrade nuclear power plant performance due to a broad range of power variations. In addition to these, uncertainties in the measurement of reactor power and neutron flux add further problems to the control design. Consequently, the traditional controllers suffer in delivering optimal performance. Thus, it is of prime importance to improve the existing control techniques for the enhanced safety and operability of a nuclear power plant.

Model Predictive Control (MPC) is an advanced control design approach that has been employed considerably in industry [1]. MPC has the ability to adapt to new operating conditions and allows simpler constraints handling. It solves an optimization problem to determine future control input over a time period. Traditionally, MPC calls for an accurate mathematical model of the process to design the control law.

Vineet Vajpayee (vineet.vajpayee@port.ac.uk), Victor Becerra (victor.becerra@port.ac.uk), and Nils Bausch (nils.bausch@port.ac.uk) are with School of Energy and Electronic Engineering, University of Portsmouth, Portsmouth, PO1 3DJ, United Kingdom.

Shohan Banerjee (s.banerjee@leedsbeckett.ac.uk) and Jiamei Deng (j.deng@leedsbeckett.ac.uk) are with School of Built Environment, Engineering, and Computing, Leeds Beckett University, Leeds, LS6 3QS, United Kingdom.

S. R. Shimjith (srshim@barc.gov.in) is with Reactor Control System Design Section, Bhabha Atomic Research Centre, Mumbai, 400 085, India and Homi Bhabha National Institute, Mumbai, 400 094, India.

A. John Arul (arul@igcar.gov.in) is with Probabilistic Safety, Reactor Shielding and Nuclear Data Section, Indira Gandhi Centre for Atomic Research, Kalpakkam, 603 102, India.
The application of MPC to the constrained load-following operation of a pressurized water-type reactor (PWR) has engaged vast research interest in the last decade [2]-[5]. For instance, $\mathrm{Na}$ et al. [2] applied MPC to the control of power level and axial power distribution. The load-following problem for movable nuclear power plants is attempted using the multiple MPC approach [3]. Eliasi et al. [4] designed a robust MPC strategy for bounding xenon oscillations. The load-following operation at a low-load working condition is studied using quadratic programming approach [5].

Although predictive controller design approaches optimize the performance of constrained systems, they lack disturbance handling capabilities. Measurements of reactor power, neutron flux, and coolant temperatures usually contain noise, uncertainties, and unmeasured disturbances. In the presence of strong disturbances, the response of predictive controller deviates far from the real output and thus the control performance can degrade greatly and may even cause system instability [6], [7].

In recent past, observer-based control approaches have been proposed to eliminate the effect of uncertainties and external disturbances. Disturbance observer (DOB) based control is one of the widely employed strategies for handling uncertainties and disturbances [8]. It utilizes the inverse model of the plant to estimate the unknown disturbances. The estimated disturbances are then combined with the control input to mitigate the effect of actual disturbances on the plant output. Due to its simplicity and effectiveness, the DOB has been integrated with MPC in the process industries in the last two decades with appilcations in grinding mill [9], [10], systems with dead-time [11], flight control [12], static var compensator [13], and selective catalytic reduction denitrification system [14]. These integrated schemes provided improved closed loop performances.

Owing to the advantages of both DOB and MPC, their combination can remove the effect of unknown disturbances and plant behaviour variations quickly while guaranteeing optimal performance. However, the performance of the DOBbased MPC control is heavily dependent on the employed model of the process. The scheme requires a priori an accurate mathematical description of the underlying process. Moreover, the model is also required to be invertible. The necessity of an accurate plant model becomes quite stringent especially when retrofitting new controllers in an aged nuclear power plant [15]. As a result, the traditional MPC-based design techniques can demonstrate sub-optimal control performance. These conditions may further increase the complexity of the overall scheme due to the conservative 
nature of constraints.

In contrast, data-based approaches such as Subspace Predictive Control (SPC) allows easy adaptation to the timevarying characteristic of the process [6], [7], [16]-[18]. SPC is a recently developed data-driven predictive control strategy based on the subspace matrix structure. It combines the estimation of a linear predictor using subspace identification with the formulation of receding horizon control design. In contrast to the traditional MPC which first models the process and then estimates the controller parameters, the SPC combines these two steps into one, thereby reducing the computation time, complexity, and errors arising due to modelplant mismatch. SPC directly designs the controller from the recorded measurement data. The formulation of SPC neither requires the solution of a non-linear Riccati equation, as is the case with linear quadratic control, nor the solution of a recursive Diophantine equation, as with generalised predictive control. The realization of SPC is through singular value and $\mathrm{QR}$ decompositions, which make the algorithm numerically stable and computationally efficient.

In this work, a data-driven Disturbance Observer-based Subspace Predictive Control (DOSPC) approach is proposed using a subspace matrix structure for the effective control of a Pressurized Water Reactor (PWR). The approach integrates a feed-forward control based on subspace-based DOB and a feedback control based on SPC. The main advantage of the proposed technique is that it can be used in the absence of a plant model. The efficacy of the proposed DOSPC is validated using two different load-following transients of a PWR. The proposed control scheme is effectively able to overcome disturbances present in the system while guaranteeing the optimal performance. The controller is suitably able to track typical ramp and step-type variations in the reactor power. The control performance of the proposed DOSPC is further compared with that of the classical SPC.

The rest of the paper is organized as follows: Section II formulates the problem. Section III presents the proposed control scheme. Section IV evaluates the proposed technique on a simulated PWR-type nuclear reactor and discusses its effectiveness through simulation results. Conclusions are drawn in Section V indicating main contributions.

\section{PROBlem Formulation}

Consider a linear uncertain systems given as,

$$
\begin{aligned}
x[k+1] & =A x[k]+B(u[k]+\xi[k]), \\
y[k] & =C x[k],
\end{aligned}
$$

where $x[k] \in \mathbb{R}^{n}, u[k] \in \mathbb{R}^{m}$, and $y[k] \in \mathbb{R}^{l}, \xi[k] \in \mathbb{R}^{m}$ represent state, input, output, and disturbance, respectively. $A \in \mathbb{R}^{n \times n}, B \in \mathbb{R}^{n \times m}$, and $C \in \mathbb{R}^{l \times n}$ are system matrices. It is assumed that $(A, B)$ is controllable and that the system uncertainties are unknown and bounded.

The problem is to design a control input $\left(\mathbf{u}_{\mathbf{f}}\right)$, such that the predicted output $\left(\hat{\mathbf{y}}_{\mathbf{f}}\right)$ tracks a reference signal $\left(\mathbf{r}_{\mathbf{f}}\right)$ in the presence of uncertainty $\xi[k]$ without violating the constraints,

$$
\begin{array}{ll}
U_{\min } \leq \mathbf{u}_{\mathbf{f}} \leq U_{\max } ; & \Delta U_{\min } \leq \Delta \mathbf{u}_{\mathbf{f}} \leq \Delta U_{\max } ; \\
Y_{\min } \leq \mathbf{y}_{\mathbf{f}} \leq Y_{\max } ; & \Delta Y_{\min } \leq \Delta \mathbf{y}_{\mathbf{f}} \leq \Delta Y_{\max } ;
\end{array}
$$

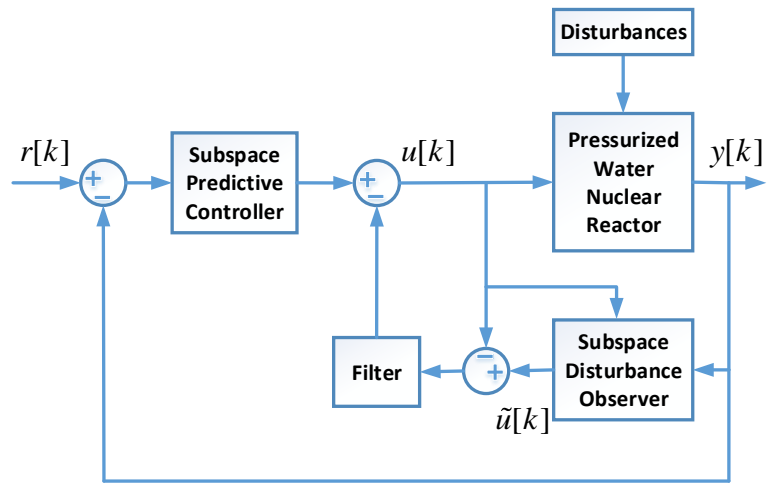

Fig. 1. Block diagram of the proposed DOSPC algorithm.

To achieve this objective the control scheme proposed in this work is depicted in Fig. 1. The control input is computed by minimizing the quadratic cost function,

$$
J=\min _{\Delta \mathbf{u}_{\mathbf{f}}}\left(\left(\hat{\mathbf{y}}_{\mathbf{f}}-\mathbf{r}_{\mathbf{f}}\right)^{T} Q_{f}\left(\hat{\mathbf{y}}_{\mathbf{f}}-\mathbf{r}_{\mathbf{f}}\right)+\Delta \mathbf{u}_{\mathbf{f}}^{T} R_{f} \Delta \mathbf{u}_{\mathbf{f}}\right),
$$

where,

$\mathbf{r}_{\mathbf{f}}=\left[\begin{array}{llll}r^{T}[k+1] & r^{T}[k+2] & \cdots & r^{T}\left[k+N_{p}\right]\end{array}\right]^{T}$, $\hat{\mathbf{y}}_{\mathbf{f}}=\left[\begin{array}{llll}\hat{y}^{T}[k+1] & \hat{y}^{T}[k+2] & \cdots & \hat{y}^{T}\left[k+N_{p}\right]\end{array}\right]^{T}$, $\mathbf{u}_{\mathbf{f}}=\left[\begin{array}{llll}u^{T}[k+1] & u^{T}[k+2] & \cdots & u^{T}\left[k+N_{c}\right]\end{array}\right]^{T}$. $N_{c}\left(\leq N_{p}\right)$ and $N_{p}$ are control and prediction horizons, respectively. $R_{f}=I_{N_{c}} \otimes R$ penalizes the rate of change of input, where $R \in \mathbb{R}^{m \times m}$ is a positive definite matrix, $I_{N_{c}}$ is an $N_{c} \times N_{c}$ identity matrix, and $\otimes$ denotes the Kronecker product. Similarly, $Q_{f}=I_{N_{p}} \otimes Q$ penalizes the error between desired reference and output, where $Q \in \mathbb{R}^{l \times l}$ is a positive semi-definite matrix.

\section{Proposed Control Approach}

\section{A. Subspace Predictor}

The formulation of SPC requires the design of a predictor to compute the control law. A set of block Hankel matrices can be formed from the collected measurement data at different equilibrium points as,

$$
\begin{aligned}
Y_{P}= & {\left[\begin{array}{cccc}
y[1] & y[2] & \cdots & y[N-2 f+1] \\
y[2] & y[3] & \cdots & y[N-2 f+2] \\
\vdots & \vdots & \ddots & \vdots \\
y[f] & y[f+1] & \cdots & y[N-f]
\end{array}\right] ; \text { (4) } } \\
Y_{F}= & {\left[\begin{array}{cccc}
y[f+1] & y[f+2] & \cdots & y[N-f+1] \\
y[f+2] & y[f+3] & \cdots & y[N-f+2] \\
\vdots & \vdots & \ddots & \vdots \\
y[2 f] & y[2 f+1] & \cdots & y[N]
\end{array}\right] \text { (5) } }
\end{aligned}
$$

where $f$ is the order of predictor matrix. $Y_{P} \in$ $\mathbb{R}^{f l \times(N-2 f+1)}$ and $Y_{F} \in \mathbb{R}^{f l \times(N-2 f+1)}$ are called the past and future output data Hankel matrices, respectively. Similarly, $U_{P} \in \mathbb{R}^{f m \times(N-2 f+1)}, E_{P} \in \mathbb{R}^{f l \times(N-2 f+1)}$, and $X_{P} \in \mathbb{R}^{n \times(N-2 f+1)}$ are defined as past input, past 
innovation, and past state matrices, respectively. The same notation holds true with subscript $F$ terms to define future Hankel matrices. Using these definitions,

$$
\begin{aligned}
& Y_{P}=\Gamma_{f} X_{P}+H_{f}^{d} U_{P}+H_{f}^{s} E_{P}, \\
& Y_{F}=\Gamma_{f} X_{F}+H_{f}^{d} U_{F}+H_{f}^{s} E_{F}, \\
& X_{F}=A^{f} X_{P}+\Delta_{f}^{d} U_{P}+\Delta_{f}^{s} E_{P},
\end{aligned}
$$

where $\Gamma_{f} \in \mathbb{R}^{f l \times n}$ is the extended observability matrix. $H_{f}^{d} \in \mathbb{R}^{f l \times f m}$ and $H_{f}^{s} \in \mathbb{R}^{f l \times f l}$ are deterministic and stochastic lower block-triangular Toeplitz matrices, respectively. $\Delta_{f}^{d} \in \mathbb{R}^{n \times f m}$ and $\Delta_{f}^{s} \in \mathbb{R}^{n \times f l}$ are deterministic and stochastic reverse extended controllability matrices, respectively. Thus, the predictor is given by

$$
\hat{Y}_{F}=L_{w} W_{P}+L_{u} U_{F},
$$

where $W_{P}=\left[\begin{array}{cc}Y_{P}^{T} & U_{P}^{T}\end{array}\right]^{T} \in \mathbb{R}^{f(m+l) \times(N-2 f+1)} . L_{w} \in$ $\mathbb{R}^{f l \times f(m+l)}$ and $L_{u} \in \mathbb{R}^{f l \times f m}$ are predictor matrices at different equilibrium points.

\section{B. Subspace Predictive Control}

In order to incorporate the above defined predictor in SPC, only the leftmost column of $\hat{Y}_{f}$ needs to be considered. Thus, (7) can be rewritten as

$$
\hat{\mathbf{y}}_{\mathbf{f}}=L_{w} \mathbf{w}_{\mathbf{p}}+L_{u} \mathbf{u}_{\mathbf{f}},
$$

or simply in terms of input increments as,

$$
\hat{\mathbf{y}}_{\mathbf{f}}=\overline{\mathrm{I}}_{l} y[k]+O_{l} L_{w} \Delta \mathbf{w}_{\mathbf{p}}+O_{l} L_{u} \Delta \mathbf{u}_{\mathbf{f}},
$$

where

$O_{l}=\left[\begin{array}{cccc}I_{l} & 0 & \cdots & 0 \\ I_{l} & I_{l} & \cdots & 0 \\ \vdots & \vdots & \ddots & \vdots \\ I_{l} & I_{l} & \cdots & I_{l}\end{array}\right] \in R^{N_{p} l \times N_{p} l}, \overline{\mathrm{I}}_{l}=\left[\begin{array}{c}I_{l} \\ I_{l} \\ \vdots \\ I_{l}\end{array}\right] \in R^{N_{p} l \times l}$,

and $I_{l}$ is an $l \times l$ identity matrix. Rewriting (9), by using $\overline{\mathbf{y}}[k]=\overline{\mathrm{I}}_{l} y[k], \bar{L}_{w}=O_{l} L_{w}$, and $\bar{L}_{u}=O_{l} L_{u}$, as

$$
\hat{\mathbf{y}}_{\mathbf{f}}=\overline{\mathbf{y}}[k]+\bar{L}_{w} \Delta \mathbf{w}_{\mathbf{p}}+\bar{L}_{u} \Delta \mathbf{u}_{\mathbf{f}} .
$$

For the case of unconstrained SPC design, the input increment can be computed as

$\Delta \mathbf{u}_{\mathbf{f}}=-\left(\left(\bar{L}_{u}\right)^{T} Q_{f} \bar{L}_{u}+R_{f}\right)^{-1}\left(\bar{L}_{u}\right)^{T} Q_{f}\left(\overline{\mathbf{y}}[k]-\mathbf{r}_{\mathbf{f}}+\bar{L}_{w} \Delta \mathbf{w}_{\mathbf{p}}\right)$,

or simply,

$$
\Delta \mathbf{u}_{\mathbf{f}}=-K_{u}\left(\overline{\mathbf{y}}[k]-\mathbf{r}_{\mathbf{f}}\right)-K_{w} \Delta \mathbf{w}_{\mathbf{p}}
$$

where the gain matrices are defined by,

$$
\begin{aligned}
& K_{u}=\left(\left(\bar{L}_{u}\right)^{T} Q_{f} \bar{L}_{u}+R_{f}\right)^{-1}\left(\bar{L}_{u}\right)^{T} Q_{f}, \\
& K_{w}=\left(\left(\bar{L}_{u}\right)^{T} Q_{f} \bar{L}_{u}+R_{f}\right)^{-1}\left(\bar{L}_{u}\right)^{T} Q_{f} \bar{L}_{w} .
\end{aligned}
$$

Finally, the control signal is updated using only the first element of the control move

$$
u[t+1]=\Delta \mathbf{u}_{\mathbf{f}}[1]+u[k] .
$$

\section{Subspace-based Disturbance Observer}

The idea of subspace-based disturbance observer is to estimate the effects of unknown disturbances and plant behaviour variations using subspace matrices. Instead of using the inverse of the plant model, the subspace predictor relation is utilized to design the subspace-based DOB. The methodology employs the inverse of subspace-based predictor to calculate the gain of the DOB and thus to estimate the effect of disturbance and uncertainness design [14].

Under the assumption that the system is of minimum phase, (8) can be rewritten as

$$
\tilde{u}[k]=\left(L_{u}^{d}\right)^{-1}\left(y[k]-L_{w}^{d} w_{p}^{d}\right)
$$

where $L_{u}^{d}$ and $L_{w}^{d}$ are the gains of the DOB and they are derived from the predictor matrices as,

$$
\begin{aligned}
& L_{u}^{d}=L_{u}(1: l,:) \\
& L_{w}^{d}=L_{w}(1: l, 1: m)
\end{aligned}
$$

The effect of unknown disturbances is reflected in the estimated control input. Thus, by subtracting it from the actual control input, the equivalent disturbance can be estimated. From Fig. 1, the estimated disturbance is given by,

$$
\hat{D}(z)=Q(z) G^{-1}(z) Y(z)-Q(z) U(z)
$$

where $G(z), Q(z), Y(z)$, and $U(z)$ denote the z-domain equivalent of PWR model, filter, output, and input, respectively. The estimated disturbance estimation is then supplied as a feed-forward signal and is subtracted from the feedback control input to compute the overall control signal to be applied to the plant. Let the output of the plant is given by,

$$
Y(z)=U(z) G(z)+D(z) G(z)
$$

From (18), the estimate of disturbance can be obtained as,

$$
\hat{D}(z)=Q(z) D(z)
$$

If the estimated disturbance is fed back to compensate the influence of the lumped disturbance, the output of the equivalent system can be given as

$$
Y(z)=U(z) G(z)-Q(z) D(z) G(z)+D(z) G(z)
$$

In another case, let the output of the plant is related as,

$$
Y(z)=U(z) G(z)+D(z)
$$

Again using (18), the estimate of disturbance is given as,

$$
\hat{D}(z)=Q(z) D(z) G^{-1}(z)
$$

Hence, the output of the equivalent system is given by

$$
Y(z)=\left(U(z)-Q(z) D(z) G^{-1}(z)\right) G(z)+D(z)
$$

It can be noted that the subspace-based DOB can estimate the unknown disturbances, (20) and (23), and the desired response, (21) and (24), can be obtained by tuning the filter. The filter $Q(z)$ is designed to be a low-pass filter with steadystate gain value of 1 [19]. Thus, the plant would behave like a nominal system with the integration of disturbance observer. 


\section{Case Study: Application to a Simulated PWR-TYPE NUCLEAR REACTOR}

The dynamical model of a PWR can be defined using the point kinetics equation with six groups of delayed neutrons precursors' concentration coupled with thermal hydraulics. The model is based on the following assumptions: the primary loop is characterized by a lumped model; the pressure and mass flow rate are constants; and the heat generated in the core is transferred using a single phase coolant. The model equations are given by:

$$
\begin{aligned}
\frac{d P}{d t} & =\frac{\rho_{T}-\sum_{i=1}^{6} \beta_{i}}{\Lambda} P+\frac{\sum_{i=1}^{6} \beta_{i} C_{i}}{\Lambda} \\
\frac{d C_{i}}{d t} & =\lambda_{i}\left(P-C_{i}\right), \quad i=1,2, \ldots, 6 \\
\frac{d T_{f}}{d t} & =H_{f} P-\gamma_{f}\left(T_{f}-T_{c}\right) \\
\frac{d T_{c}}{d t} & =-H_{c}\left(T_{\text {out }}-T_{\text {in }}\right)+\gamma_{c}\left(T_{f}-T_{c}\right), \\
\frac{d T_{\text {in }}}{d t} & =\frac{1}{\tau_{\text {cold }}}\left(D_{2} T_{s g}-D_{3} T_{h o t}-T_{i n}\right) \\
\frac{d T_{h o t}}{d t} & =\frac{1}{\tau_{h o t}}\left(T_{\text {out }}-T_{h o t}\right) \\
\frac{d T_{s g}}{d t} & =-\frac{1}{\tau_{s g}}\left(T_{s g}-T_{h o t}\right)-D_{1} L_{T} \\
T_{c} & =\frac{T_{\text {out }}+T_{\text {in }}}{2} \\
\rho_{T} & =\rho+\alpha_{f} T_{f}+\alpha_{c} T_{c}
\end{aligned}
$$

where $P$ is normalized neutronic power; $\beta_{i}, \lambda_{i}$, and $C_{i}$ denote fraction of delayed neutrons, decay constant, and normalized delayed neutron precursors' concentration of $i^{t h}$ group, respectively; $\Lambda$ represents prompt neutron life time; $T_{f}$ and $T_{c}$ are temperatures of fuel and coolant, respectively; $\alpha_{f}$ and $\alpha_{c}$ are temperature coefficients of reactivity of fuel and coolant, respectively; $H_{f}, H_{c}, \gamma_{f}$, and $\gamma_{c}$ are proportionality constants; $T_{\text {out }}, T_{\text {in }}, T_{\text {hot }}$, and $T_{s g}$ are temperatures of core-outlet, core-inlet, hot leg and steam generator, respectively. $\tau_{\text {cold }}, \tau_{h o t}$, and $\tau_{s g}$ are time constants; $D_{1}$, $D_{2}$, and $D_{3}$ are constants; $L_{T}$ is turbine load; $\rho$ and $\rho_{T}$ denote reactivity contributed by control input and the total reactivity, respectively; It is assumed that initially the reactor is operating at 0.5 fractional full power (FFP). Values of various parameters used in (25)-(33) at 0.5 FFP are listed in Table I [3], [7]. The system given by (25)-(33) is perturbed by a reactivity transient to obtain the corresponding variation in reactor power. The reactivity and power are considered as input and output of the reactor system, respectively and thus form the estimation dataset for designing the nominal control.

\section{A. Case Study I}

A study is carried out to examine the step and ramp type disturbances handling capability of the proposed controller for the following load-following mode of operation:

$$
P= \begin{cases}0.50 P, & 0 s \leq t \leq 1.8 s ; \\ 0.5(t-1.8) / 60+0.50, & 1.8 s<t \leq 3.6 s ; \\ 0.515 P, & 3.6 s<t \leq 5.4 s ; \\ 0.5(t-5.4) / 60+0.515, & 5.4 s<t \leq 7.2 s \\ 0.53 P, & 7.2 s<t \leq 9.0 s \\ 0.5(t-9.0) / 60+0.53, & 9.0 s<t \leq 9.72 s ; \\ 0.536 P, & 9.72 s<t \leq 12.6 s \\ -0.5(t-12.6) / 60+0.536, & 12.6 s<t \leq 13.32 s ; \\ 0.53 P, & 13.32 s<t \leq 15.12 s ; \\ -0.5(t-15.12) / 60+0.53, & 15.12 s<t \leq 16.92 s \\ 0.515 P, & 16.92 s<t \leq 18.72 s \\ -0.5(t-18.72) / 60+0.515, & 18.72 s<t \leq 20.52 s \\ 0.50 P, & 20.52 s<t \leq 24.12 s \\ 0.53 P, & 24.12 s<t \leq 27.72 s \\ 0.50 P, & 27.72 s<t \leq 35 s ;\end{cases}
$$

A step and ramp type disturbance is applied to the incremental change of reactivity as

$\Delta \xi(t)= \begin{cases}-1 \times 10^{-7}, & 7.2 s<t \leq 9.0 s \\ 1 \times 10^{-7}(t-14.4), & 14.4 s<t \leq 22.5 s ; \\ 1 \times 10^{-7}, & 28.8 s<t \leq 29.7 s\end{cases}$

Performance of the proposed DOSPC controller and that of the classical SPC controller for tracking the reference power in the presence of disturbances are shown in Fig. 2. It can be observed that the proposed DOSPC is able to track the reference set-point better than that of the classical SPC. DOSPC can remove the effect of step and ramp disturbances. Variations of control signal and rate of change of control signal are shown in Figs. 3 and 4, respectively. Variations of actual and estimated disturbances are shown in Fig. 5.

\section{B. Case Study II}

Another load-following mode of operation is considered to validate the effectiveness of the proposed controller during large variation in power as follows:

$$
P= \begin{cases}0.50 P, & 0 s \leq t \leq 18 s \\ 0.60 P, & 18 s<t \leq 54 s \\ 0.50 P, & 54 s<t \leq 90 s \\ -1.0(t-90) / 6+0.50, & 90 s<t \leq 144 s \\ 0.41 P, & 144 s<t \leq 180 s \\ 1.0(t-180) / 6+0.41, & 180 s<t \leq 234 s \\ 0.50 P, & 234 s<t \leq 270 s \\ 0.60 P, & 270 s<t \leq 306 s \\ 0.50 P, & 306 s<t \leq 350 s\end{cases}
$$

A sinusoidal disturbance is applied to the reactivity input as,

$$
\xi(t)=\left\{\begin{array}{l}
1 \times 10^{-2} \sin (5 t), 36 s<t \leq 180 s \\
2 \times 10^{-2} \sin (2 t), 180 s<t \leq 324 s
\end{array}\right.
$$

Performance of the proposed DOSPC controller and that of the classical SPC controller for tracking the reference are shown in Fig. 6. It can be observed that the proposed DOSPC is able to track the reference set-point better than that of the classical SPC. DOSPC can remove the effect of sinusoidal 
TABLE I

NEUTRONIC AND THERMAL-HYDRAULIC PARAMETERS

\begin{tabular}{|c|c|c|c|c|c|c|}
\hline Group, i & 1 & 2 & 3 & 4 & 5 & 6 \\
\hline$\lambda_{i}\left(s^{-1}\right)$ & 0.0125 & 0.0308 & 0.1152 & 0.3109 & 1.240 & 3.3287 \\
\hline$\beta_{i}$ & 0.000216 & 0.001416 & 0.001349 & 0.00218 & 0.00095 & 0.000322 \\
\hline \hline$H_{f}\left({ }^{\circ} \mathrm{Cs}^{-1}\right)$ & $H_{c}\left(s^{-1}\right)$ & $\gamma_{f}\left(s^{-1}\right)$ & $\gamma_{c}\left(s^{-1}\right)$ & $\alpha_{f}\left({ }^{\circ} \mathrm{C}^{-1}\right)$ & $\alpha_{c}\left({ }^{\circ} \mathrm{C}^{-1}\right)$ & $\Lambda(s)$ \\
22.22 & 1.0261 & 0.1751 & 0.0571 & $-2.9 \times 10^{-5}$ & $-6.3 \times 10^{-4}$ & $1.76 \times 10^{-4}$ \\
\hline$\tau_{\text {cold }}(s)$ & $\tau_{\text {hot }}(s)$ & $\tau_{s g}(s)$ & $D_{1}\left({ }^{\circ} \mathrm{Cs}^{-1}\right)$ & $D_{2}$ & \multicolumn{2}{|c|}{$D_{3}$} \\
7.0 & 5.0 & 11.3 & 3.746 & 0.7005 & \multicolumn{2}{|c|}{-0.2995} \\
\hline
\end{tabular}

TABLE II

COMPARISON OF PERFORMANCE OF DIFFERENT APPROACHES

\begin{tabular}{|c|c|c|c|c|}
\hline Case & Technique & PRMSE & TVI & $\|U\|_{2}$ \\
\hline \multirow{2}{*}{ I } & DOSPC & $1.084 \times 10^{-3}$ & $1.078 \times 10^{-3}$ & $3.112 \times 10^{-2}$ \\
\cline { 2 - 5 } & SPC & $1.193 \times 10^{-3}$ & $1.398 \times 10^{-3}$ & $4.128 \times 10^{-2}$ \\
\hline \multirow{2}{*}{ II } & DOSPC & $4.848 \times 10^{-4}$ & $6.644 \times 10^{-3}$ & $2.567 \times 10^{-1}$ \\
\cline { 2 - 5 } & SPC & $5.204 \times 10^{-4}$ & $1.101 \times 10^{-2}$ & $3.855 \times 10^{-1}$ \\
\hline
\end{tabular}

disturbances. Variations of control signal and rate of change of control signal are shown in Figs. 7 and 8, respectively. Variations of incremental change of actual and estimated disturbances are shown in Fig. 9.

\section{Performance Assessment}

A quantitative performance assessment is conducted by analysing the effects of control actions on the output and the input. Performance measures such as, percentage root mean squared error (PRMSE) computed between output and demand set-point, total variation of input (TVI), and the L2norm of input $\left(\|U\|_{2}\right)$ are computed. These are given by,

$$
\begin{aligned}
\text { PRMSE } & =\frac{1}{N} \sqrt{\sum_{k=1}^{N}(y[k]-r[k])^{2}} \times 100 \%, \\
T V I & =\sum_{k=1}^{N}|(u[k+1]-u[k])| \\
\|U\|_{2} & =\sqrt{\left(\sum_{k=1}^{N}(u[k])^{2}\right)} .
\end{aligned}
$$

Table II compares the control performances of DOSPC and SPC approaches. It is found that the values of PRMSE, TVI, and the $\|U\|_{2}$ for DOSPC approaches is less than that of the classical SPC approach in both the cases. The controller provides better set point tracking with less control efforts in the presence of disturbances. Thus, it can be concluded that the DOSPC can track the demand variation better than that of the classical SPC approach.

\section{Conclusions}

A data-driven disturbance observer-based subspace predictive control approach is designed to control a PWRtype nuclear reactor. The proposed controller is realized by integrating a feed-forward control based on subspace-based DOB and a feedback control based on SPC. The controller is implemented to study two different load-following operations in a PWR. Simulation results demonstrate that the proposed control strategy maintains the desired performance. It is effectively able to overcome different disturbances present in

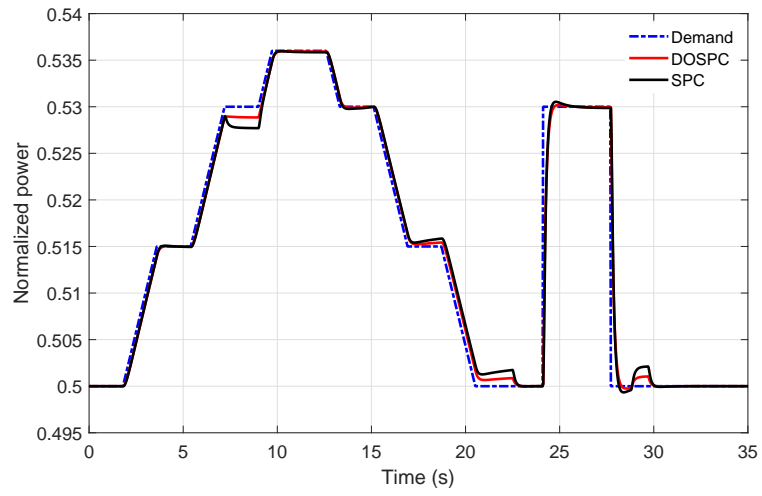

Fig. 2. Variation of reactor power for DOSPC and SPC.

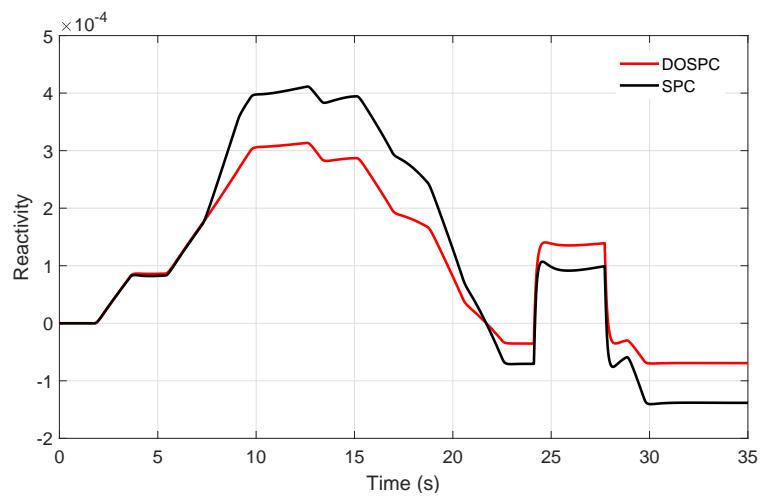

Fig. 3. Variation of control input for DOSPC and SPC.

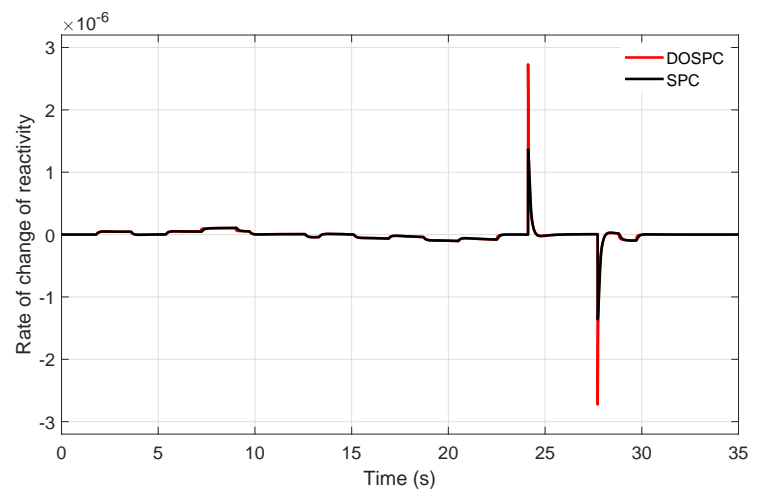

Fig. 4. Variation of rate of change of control input for DOSPC and SPC.

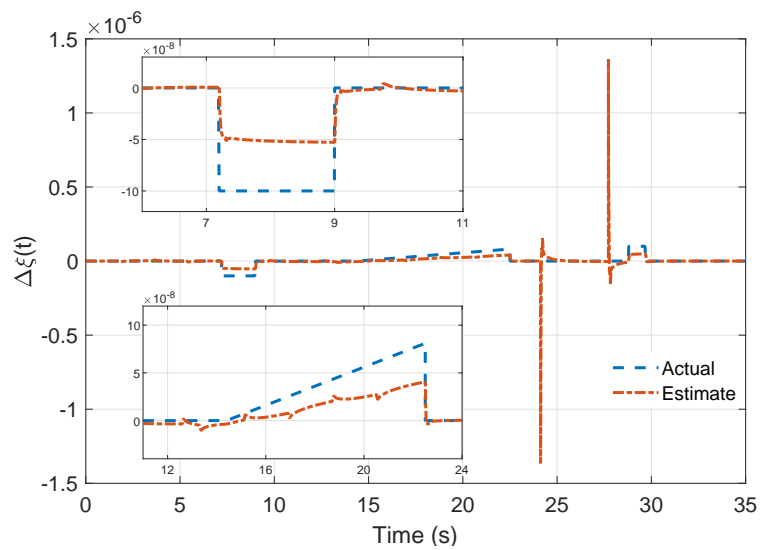

Fig. 5. Variation of actual and estimated disturbances. 


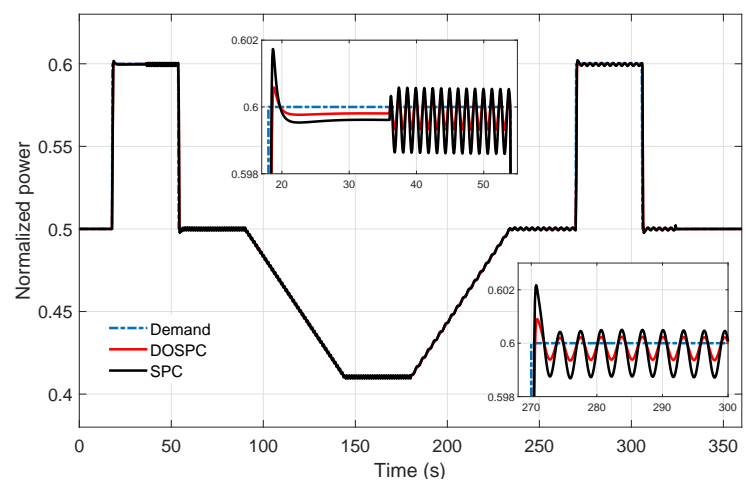

Fig. 6. Variation of reactor power for DOSPC and SPC.

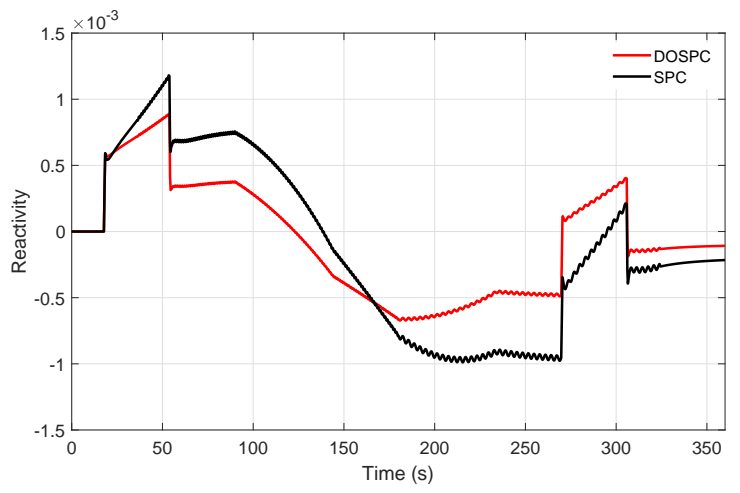

Fig. 7. Variation of control input for DOSPC and SPC.

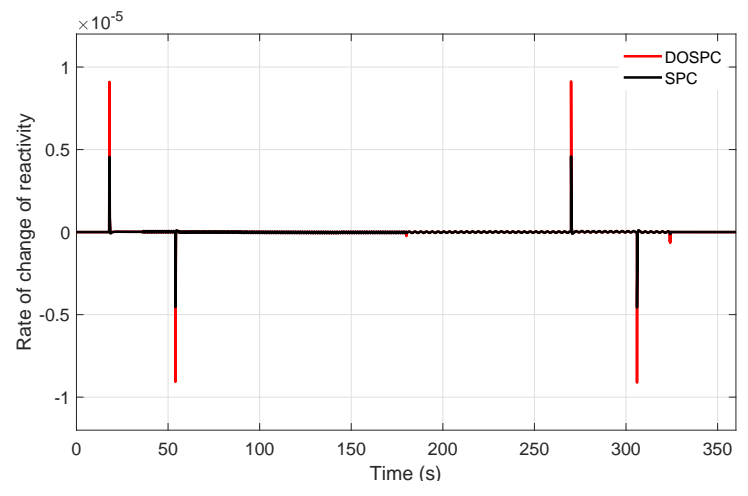

Fig. 8. Variation of rate of change of control input for DOSPC and SPC.

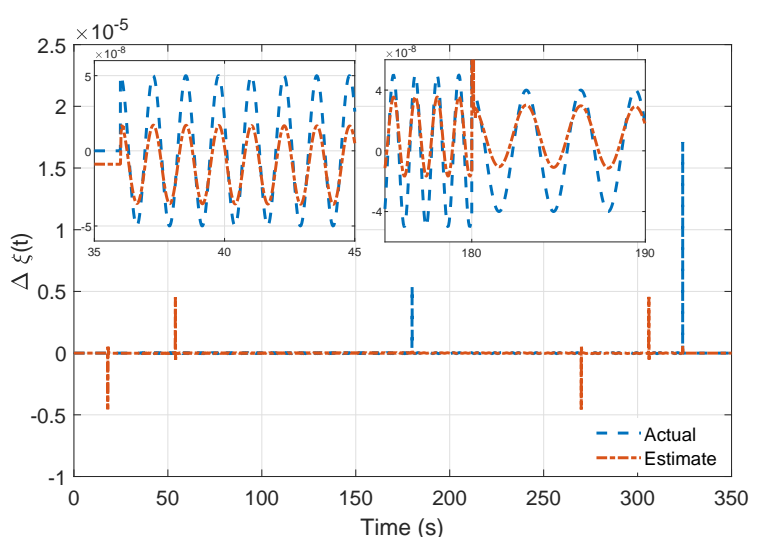

Fig. 9. Variation of incremental change of actual and estimated disturbances. the system. The control performance of the proposed DOSPC is found to be better than that of the classical SPC.

\section{ACKNOWLEDGEMENT}

The work presented in this paper has been financially supported under grants EP/R021961/1 and EP/R022062/1 from the Engineering and Physical Sciences Research Council.

\section{REFERENCES}

[1] S. J. Qin and T. A. Badgwell, "A survey of industrial model predictive control technology," Control Engineering Practice, vol. 11, no. 7, pp. 733-764, Jul 2003.

[2] M. G. Na, D. W. Jung, S. H. Shin, J. W. Jang, K. B. Lee, and Y. J. Lee, "A model predictive controller for load-following operation of PWR reactors," IEEE Transactions on Nuclear Science, vol. 52, no. 4, pp. 1009-1020, Aug 2005.

[3] T. Yun, H. Su-xia, L. Chong, and Z. Fu-yu, "An improved implicit multiple model predictive control used for movable nuclear power plant," Nuclear Engineering and Design, vol. 240, pp. 3582-3585, 2010.

[4] H. Eliasi, M. B. Menhaj, and H. Davilu, "Robust nonlinear model predictive control for nuclear power plants in load following operations with bounded xenon oscillations," Nuclear Engineering and Design, vol. 241, pp. 533-543, 2011.

[5] G. Wang, J. Wu, B. Zeng, Z. Xu, W. Wu, and X. Ma, "Statespace model predictive control method for core power control in pressurized water reactor nuclear power stations," Nuclear Engineering and Technology, vol. 49, no. 1, pp. 134-140, 2017.

[6] V. Vajpayee, S. Mukhopadhyay, and A. P. Tiwari, "Subspace-based wavelet preprocessed data-driven predictive control," INCOSE International Symposium, vol. 26, no. s1, pp. 357-371, 2016.

[7] —-, "Data-driven subspace predictive control of a nuclear reactor," IEEE Transactions on Nuclear Science, vol. 65, no. 2, pp. 666-679, Feb 2018.

[8] W. Chen, J. Yang, L. Guo, and S. Li, "Disturbance-observer-based control and related methodsan overview," IEEE Transactions on Industrial Electronics, vol. 63, no. 2, pp. 1083-1095, Feb 2016.

[9] X. Chen, J. Yang, S. Li, and Q. Li, "Disturbance observer based multivariable control of ball mill grinding circuits," Journal of Process Control, vol. 19, no. 7, pp. 1205 - 1213, 2009.

[10] J. Yang, S. Li, X. Chen, and Q. Li, "Disturbance rejection of ball mill grinding circuits using DOB and MPC," Powder Technology, vol. 198, no. 2, pp. $219-228,2010$.

[11] P. Zhou, T. Chai, and J. Zhao, "DOB design for nonminimum-phase delay systems and its application in multivariable mpc control," IEEE Transactions on Circuits and Systems II: Express Briefs, vol. 59, no. 8, pp. 525-529, Aug 2012.

[12] C. Liu, W.-H. Chen, and J. Andrews, "Tracking control of small-scale helicopters using explicit nonlinear MPC augmented with disturbance observers," Control Engineering Practice, vol. 20, no. 3, pp. 258 268, 2012.

[13] J. Yang and W. X. Zheng, "Offset-free nonlinear mpc for mismatched disturbance attenuation with application to a static var compensator," IEEE Transactions on Circuits and Systems II: Express Briefs, vol. 61, no. 1, pp. 49-53, Jan 2014.

[14] X. Wu, J. Shen, S. Sun, Y. Li, and K. Y. Lee, "Data-driven disturbance rejection predictive control for SCR denitrification system," Industrial \& Engineering Chemistry Research, vol. 55, no. 20, pp. 5923-5930, 2016.

[15] H. Eliasi, M. B. Menhaj, and H. Davilu, "Robust nonlinear mode predictive control for a PWR nuclear power plant," Progress in Nuclear Energy, vol. 54, pp. 177-185, 2012.

[16] W. Favoreel and B. D. Moor, "SPC: Subspace predictive control," in Proceedings of the IFAC World Congress, Beijing, 1998, pp. 235-240.

[17] R. Kadali, "A data driven subspace approach to predictive controller design," Control Engineering Practice, vol. 11, no. 3, pp. 261-278, 2003.

[18] X. Wu, J. Shen, Y. Li, and K. Y. Lee, "Data-driven modeling and predictive control for boiler-turbine unit," IEEE Transactions on Energy Conversion, vol. 28, no. 3, pp. 470-481, Sep 2013.

[19] H. Shim and N. H. Jo, "An almost necessary and sufficient condition for robust stability of closed-loop systems with disturbance observer," Automatica, vol. 45, no. 1, pp. 296 - 299, 2009. 